\title{
Double-pulse Brillouin distributed optical fiber sensors: analytical model and experimental validation
}

\author{
Marcelo A. Soto*a, Sanghoon Chin ${ }^{\mathrm{b}}$, Luc Thévenaz ${ }^{\mathrm{a}}$ \\ ${ }^{a}$ EPFL Swiss Federal Institute of Technology, Institute of Electrical Engineering, SCI STI LT, \\ Station 11, CH-1015 Lausanne, Switzerland; \\ ${ }^{\mathrm{b}}$ Omnisens SA, Riond-Bosson 3, CH-1110 Morges, Switzerland \\ *E-mail: marcelo.soto@epfl.ch
}

\begin{abstract}
An analytical model is presented to describe the behavior of the acoustic wave, probe signal and Brillouin gain in double-pulse Brillouin optical time-domain analysis (DP-BOTDA) sensors. The proposed model is a tool that provides a full physical insight into the Brillouin interaction occurring in this double-pulse configuration, and allows the optimization and complete analysis of the system. The proposed solution is experimentally validated in a long-range system, which is optimized to demonstrate experimentally, for the first time, the capability of DP-BOTDA to achieve a $11 \mathrm{~km}$ sensing distance with $20 \mathrm{~cm}$ spatial resolution and a frequency resolution of $0.5 \mathrm{MHz}$.
\end{abstract}

Keywords: Stimulated Brillouin scattering, distributed optical fiber sensor, temperature sensing, strain sensing

\section{INTRODUCTION}

During the last years the sensing capability of distributed optical fiber sensors to measure a physical variable along an optical fiber has attracted the attention of many industrial sectors. In particular, distributed strain and temperature sensing using stimulated Brillouin scattering (SBS) in a pump-probe configuration ${ }^{1}$ has been widely studied in both academia and industry. Unfortunately, using the standard time-domain approach, so-called Brillouin optical time-domain analysis (BOTDA) method, the best attainable spatial resolution is limited down to 1 meter, due to the response time of the acoustic waves involved in the SBS process. In order to achieve sub-meter resolution, several configurations have been proposed for the past decade. For instance, methods such as differential pulse-width pair BOTDA (DPP-DOTDA) ${ }^{2}$, Brillouin echoes (BEDS) ${ }^{3}$, pulse pre-pump BOTDA (PPP-BOTDA) ${ }^{4}$ or double-pulse BOTDA (DP-BOTDA) $)^{5}$ have been demonstrated to be suitable methods to achieve sub-meter resolution, reducing or even cancelling the well-known broadening of the Brillouin gain spectrum (BGS) occurring when short pulses $(<10 \mathrm{~ns})$ are concerned.

In particular, DP-BOTDA combines two pulses, which are sequentially launched into the fiber with a time difference shorter than the acoustic wave decay time, producing an interference pattern that narrows the actual Brillouin gain spectral response and increases the signal-to-noise ratio (SNR) of the measurement ${ }^{5}$. Nevertheless, the use of DPBOTDA to achieve centimeter-scale resolution has only been studied through numerical simulations based on the threewave SBS model ${ }^{5}$, and hence, to the best of our knowledge, the technique has never been experimentally implemented to achieve sub-meter resolution over long distances. In order to optimize the parameters of the pulses (such as pulse width and pulses interval) and to provide a better insight of the SBS process taking place in this double-pulse configuration, an analytical mathematical model could be of great interest. In Ref. 6 an analytical solution of the three-wave SBS equations has been proposed for a single pulse composed of three sections (the first section taking place from $t=-\infty$ to $t=0$, the second one from $t=0$ to $t=T$, and the third section from $t=T$ to $t=\infty$ ). Even though such a model properly describes the SBS process in all single pulse schemes, it does not cover the double-pulse configuration, and therefore, a dedicated analytical model could be a useful tool to analyze, design and optimize DP-BOTDA systems.

In this paper we present an analytical model that describes the behavior of the acoustic wave, probe signal amplitude and Brillouin gain in DP-BOTDA systems, considering arbitrary pulse widths and intervals. The validation of the model is carried out in an $11 \mathrm{~km}$-long DP-BOTDA sensor, which is designed and analyzed with the proposed model. Thus, with the optimized double-pulse configuration, we demonstrate, for the first time to our knowledge, distributed measurements along an $11 \mathrm{~km}$ using a DP-BOTDA sensor with spatial / frequency resolution of $20 \mathrm{~cm} / 0.5 \mathrm{MHz}$. 


\section{COMPLETE ANALYTICAL MODEL FOR DOUBLE-PULSE BOTDA SENSORS}

In order to obtain an analytical expression that entirely describes the acoustic wave and variations of the probe signal amplitude in a DP-BOTDA configuration, the three-wave coupled equations describing SBS have to be solved ${ }^{6}$ :

$$
\left(\frac{\partial}{\partial z}+\frac{1}{v_{g}} \frac{\partial}{\partial t}\right) E_{P}=i \frac{1}{2} g_{2} Q E_{S},
$$

$$
\left(-\frac{\partial}{\partial z}+\frac{1}{v_{g}} \frac{\partial}{\partial t}\right) E_{S}=i \frac{1}{2} g_{2} Q^{*} E_{P},
$$

$$
\left(\frac{\partial}{\partial t}+\Gamma_{A}\right) Q=i g_{1} E_{P} E_{S}^{*},
$$

where $E_{P}, E_{S}$ and $Q$ are the normalized slowly-varying envelopes of the pump, signal (probe) and acoustic fields; $v_{g}$ is the group velocity; $g_{1,2}$ are the electrostriction and elasto-optic coupling coefficients; and $\Gamma_{A}=i\left(\Omega_{B}{ }^{2}-\Omega^{2}-i \Omega \Gamma_{B}\right) / 2 \Omega$ is the local frequency detuning parameter, where $\Gamma_{B}$ is the acoustic damping constant, $\Omega / 2 \pi$ is the pump-probe frequency detuning and $\Omega_{B} / 2 \pi$ is the local Brillouin frequency shift (BFS).

Equations (1)-(3) are solved by a perturbation method in which the probe signal $E_{s}(z, t)$ is assumed in the small gain approximation to be composed of a constant component $E_{s}{ }^{0}$ and a small varying component $e_{s}(z, t)$, so that $E_{s}(z, t)=E_{s}{ }^{0}+e_{s}(z, t)$. The acoustic wave $Q(z, t)$ and variations of the signal $e_{s}(z, t)$ are analytically obtained using Laplace transform, following a similar procedure as presented in Ref. 6, but considering a double-pulse pump signal, as illustrated in Fig. 1. Thus, the following general mathematical expression for the pump signal $E_{P}(z, t)$ is used to solve Eqs. (1)-(3):

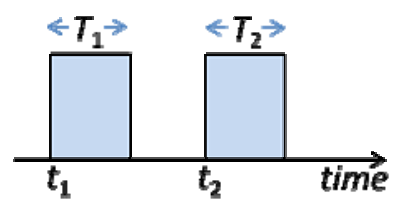

Figure 1. Double-pulse pump configuration for DP-BOTDA.

$$
E_{P}(z, t)=E_{P}^{0}\left\{u\left(t-t_{1}-z / v_{g}\right)-u\left(t-t_{1}-T_{1}-z / v_{g}\right)+u\left(t-t_{2}-z / v_{g}\right)-u\left(t-t_{2}-T_{2}-z / v_{g}\right)\right\},
$$

where $E_{p}{ }^{0}$ is the peak amplitude of the pump field, $t_{l, 2}$ are the time corresponding to the beginning of the first and second pulses, $T_{1,2}$ are the respective pulse width, and $\mathrm{u}(\cdot)$ is the Heaviside step function.

Using the undepleted pump approximation, the solution of Eq. (1) turns out to be trivial, resulting in a simple invariant translation only scaled by the term $t-z / v_{g}$. Then, using Laplace transform in Eqs. (3)-(4), the acoustic field is obtained:

$$
\begin{aligned}
Q(z, t) & =\frac{i g_{1} E_{S}^{0^{*}} E_{P}^{0}}{\Gamma_{A}}\left\{\left[1-\exp \left(-\Gamma_{A}^{*}\left(t-t_{1}-z / v_{g}\right)\right)\right] u\left(t-t_{1}-z / v_{g}\right)-\left[1-\exp \left(-\Gamma_{A}^{*}\left(t-t_{1}-T_{1}-z / v_{g}\right)\right)\right] u\left(t-t_{1}-T_{1}-z / v_{g}\right)\right. \\
& \left.+\left[1-\exp \left(-\Gamma_{A}^{*}\left(t-t_{2}-z / v_{g}\right)\right)\right] u\left(t-t_{2}-z / v_{g}\right)-\left[1-\exp \left(-\Gamma_{A}^{*}\left(t-t_{2}-T_{2}-z / v_{g}\right)\right)\right] u\left(t-t_{2}-T_{2}-z / v_{g}\right)\right\} .
\end{aligned}
$$

Hence, using Eq. (5) and Laplace transform in Eq. (2), the variations of the probe signal in time and distance, $e_{s}(z, t)$, can be obtained at a specific fiber location $z=z_{0}$, for a very short fiber section $\Delta z$ where the SBS interaction takes place:

$$
\begin{aligned}
e_{S}(z, t) & =\frac{E_{S}^{0^{*}} I_{P}^{0} g_{2} g_{1}^{0} \Delta z}{2 \Gamma_{A}^{*}}\left\{[ 1 - \operatorname { e x p } ( - \Gamma _ { A } ^ { * } ( t - t _ { 1 } - ( 2 z _ { 0 } - z ) / v _ { g } ) ) ] \cdot \left[u\left(t-t_{1}-\left(2 z_{0}-z\right) / v_{g}\right)-u\left(t-t_{1}-T_{1}-\left(2 z_{0}-z\right) / v_{g}\right)\right.\right. \\
& \left.+u\left(t-t_{2}-\left(2 z_{0}-z\right) / v_{g}\right)-u\left(t-t_{2}-T_{2}-\left(2 z_{0}-z\right) / v_{g}\right)\right]+\left[\exp \left(-\Gamma_{A}^{*}\left(t-t_{1}-T_{1}-\left(2 z_{0}-z\right) / v_{g}\right)\right)\right. \\
& \left.\left.-\exp \left(-\Gamma_{A}^{*}\left(t-t_{2}-\left(2 z_{0}-z\right) / v_{g}\right)\right)\right] \cdot\left[u\left(t-t_{2}-\left(2 z_{0}-z\right) / v_{g}\right)-u\left(t-t_{2}-T_{2}-\left(2 z_{0}-z\right) / v_{g}\right)\right]\right\} .
\end{aligned}
$$

Equations (5)-(6) describe explicitly the behavior of the acoustic wave and probe amplitude variations resulting from the double-pulse pump (including arbitrary pulse widths). The analytical solution provides a clear physical insight of the SBS process for this BOTDA configuration, allowing the analysis and design of DP-BOTDA sensors.

Note that, although, Eq. (6) describes the probe amplitude variations in a short fiber section, in case of long sensing fiber, the resulting probe signal can be simply obtained from the sum of independent $e_{s}(z, t)$ calculated at each fiber section $\Delta z$.

\section{EXPERIMENTAL VALIDATION AND DEMONSTRATION OF LONG-RANGE SENSING}

In order to validate the proposed analytical model, we compare simulations with experimental results in a long-range DP-BOTDA sensor, using a setup similar to the one reported in Ref. 7 for a standard BOTDA. The main difference in this scheme is that the pump signal is composed of two short pulses, instead of a standard long single pulse 5 . The time interval between pulses should be within the acoustic wave decay time, so that the acoustic waves generated by both pulses are combined, generating an interference pattern that narrows the BGS despite of the use of short pulses. 
Aiming at $20 \mathrm{~cm}$ spatial resolution, we have first calculated the response of a DP-BOTDA system using two pulses of $2 \mathrm{~ns}$, and separated by $\tau=t_{2}$ $t_{l}$, which has been varied from $3 \mathrm{~ns}$ up to $7 \mathrm{~ns}$. To provide a better understanding of the SBS process in this case, Fig. 2(a) shows the broadspectrum acoustic wave resulting from only one $2 \mathrm{~ns}$ pulse, and then the longer decay time after the passage of the pulse (including off-resonance oscillations). Then, the passage of second pulse generates a similar acoustic wave; however, since the pulse separation is shorter than the acoustic wave decay time, both acoustic fields superimpose, resulting in the acoustic field depicted in Fig. 2(b) (in the figure, $\tau=4 \mathrm{~ns}$ ). As we can see, the superimposition generates a pattern of destructive and constructive interferences that depends on the frequency detuning with respect to the peak gain. Fig. 3 illustrates the BGS resulting from the double-pulse configuration for several time intervals $\tau$. We can clearly observe that for the resonance frequency $\left(f=f_{B}=\mathrm{BFS}=10.47 \mathrm{GHz}\right)$, the in-phase generation of the acoustic waves leads to a constructive interference, while the acoustic fields interact destructively when the phase difference is $\pi$. Thus, for instance in case a pulse interval of $\tau=4 \mathrm{~ns}$, the first destructive interference occurring in the frequencydomain is at $f_{B}+125 \mathrm{MHz}$ (i.e. $2 \pi \times 4 \mathrm{~ns} \times 125 \mathrm{MHz}=\pi$ ) and the peak of the first sidelobes (constructive interference) takes place when the phase difference is $2 \pi$, i.e at $f_{B}+250 \mathrm{MHz}(2 \pi \times 4 \mathrm{~ns} \times 250 \mathrm{MHz}=2 \pi)$. We can observe that with longer intervals $\tau$ the main BGS lobe narrows, while the amplitude of the sidelobes increases due to the weaker interaction among both acoustic fields. We have found that a good trade-off between spectral narrowing and maximum acoustic-wave interaction occurs when the amplitude of the sidelobes is equal to half of the amplitude of the main lobe. This condition occurs when the pulse interval is about $\tau=4 \mathrm{~ns}$; and therefore, we have experimentally tested our model under such a pulse configuration. To evaluate this scheme, the parameters of the pump in Fig. 1 and Eq. (1) are: $t_{1}=5 \mathrm{~ns}, t_{2}=9 \mathrm{~ns}$, and $T_{1}=T_{2}=2 \mathrm{~ns}$.
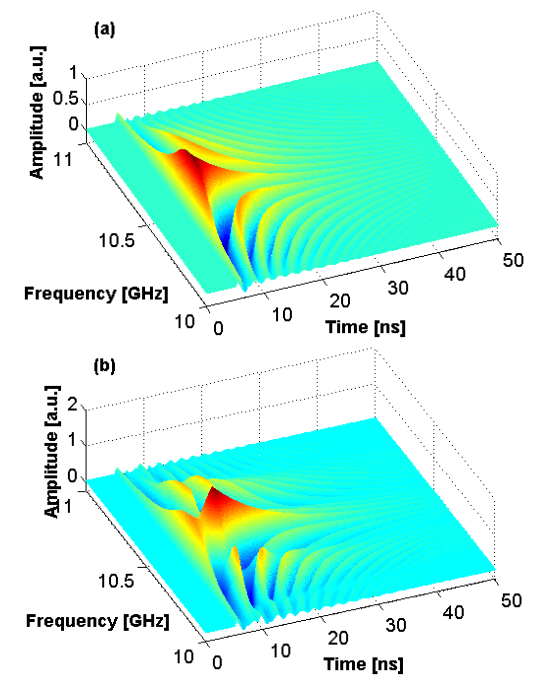

Figure 2. Acoustic wave created in SBS process when using (a) a single 2 ns pulse,

(b) two 2 ns pulses separated by $\tau=2$ ns.

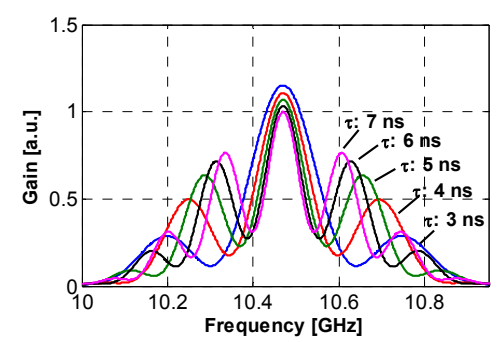

Figure 3. BGS narrowing in a DP-BOTDA sensor as a function of the pulse separation $\tau$.

Figures 4(a)-(b) show the BGS along the first few meters of fiber as a function of time and frequency. In particular Fig. 4(a) presents the calculated results while Fig. 4(b) reports the respective measured BGS. We can observe in both figures a first broad gain spectrum generated by the first $2 \mathrm{~ns}$ pulse (which enters into the fiber at $t=5 \mathrm{~ns}$ ), and then the expected interference pattern occurring when the second pump pulse enters into the fiber at $t=9 \mathrm{~ns}$. For a clearer comparison, a top-view of the respective BGS is shown in Fig. 4(c) (analytical calculation) and Fig. 4(d) (experiment). We can clearly see the good agreement between both the analytical results and the experimental data.
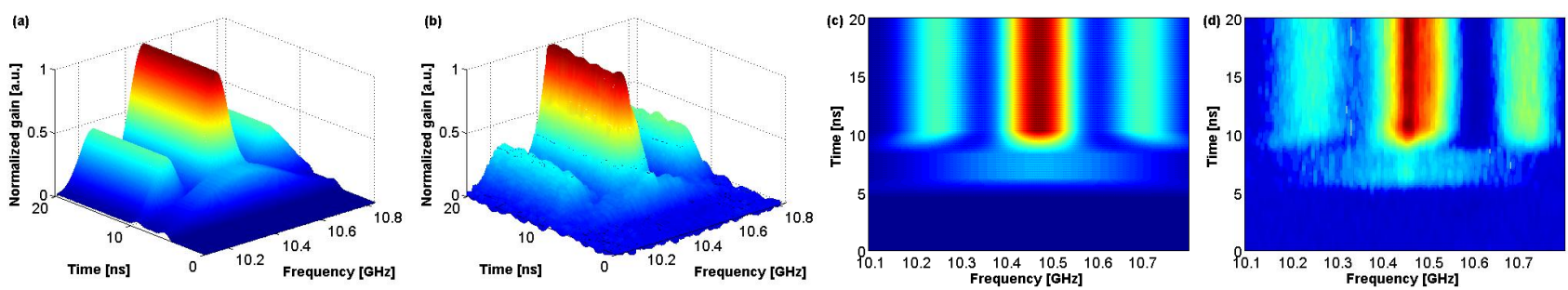

Figure 4. Brillouin gain spectrum along the first meters of fiber. (a) Simulated, and (b) measured spectrum. The respective top-views are shown in (c) simulations and (d) measured spectrum.

If we consider that at the resonance frequency the optical fields generated by both the acoustic waves superpose constructively, the resulting power variation of the probe signal, measured at the receiver, is four-fold higher than the intensity contrast obtained by a single short pulse. This feature gives a significant advantage to the use of double-pulse configuration, since the system benefits from the quadratic detection of the optical intensity rather than being proportional to the optical field amplitude. This behavior can also be observed in the analytical results, which can be fairly compared to the measured trace, as evidenced in Fig 5. 
With the optimized double-pulse configuration we have carried out distributed measurements along a $11 \mathrm{~km}$-long dispersion shifted fiber (using 1000 time-averaged traces), obtaining a uniform pattern of interference in the measured BGS, as shown in Fig. 6(a). The BGS measured at $1 \mathrm{~km}$ distance has been compared in Fig. 6(b) to the analytically calculated spectrum. Although a slight asymmetry in the measured BGS can be observed (due to potential variations of the probe $\mathrm{CW}$ power as function of the frequency, resulting from the double sideband modulation; for more details refer to the setup in Ref. 7), a good agreement between experimental data and the analytical expression can be verified.

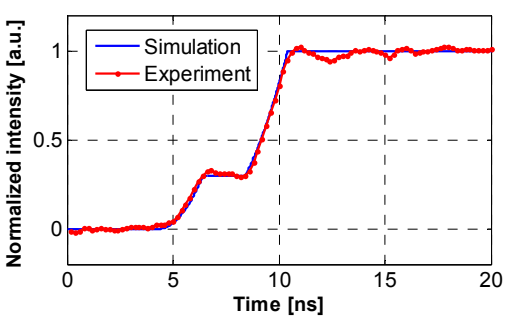

Figure 5. Probe intensity in the first meters of fiber at resonance $\left(f=f_{B}\right)$.
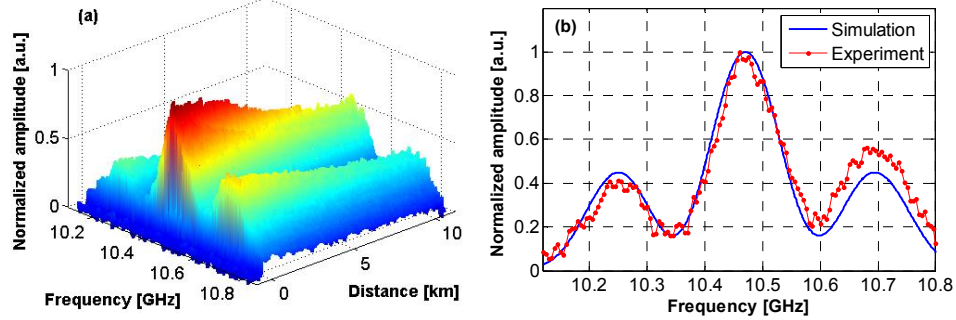

Figure 6. (a) BGS measured along an $11 \mathrm{~km}$ fiber. (b) Comparison of simulated and measured BGS at $1 \mathrm{~km}$ distance

Finally, in order to demonstrate the real spatial resolution of the implemented system, a hot-spot of $20 \mathrm{~cm}$, and $10^{\circ} \mathrm{C}$ difference with respect to room temperature, has been measured near the fiber end (at $\sim 11 \mathrm{~km}$ distance). Figure 7 shows both the BFS obtained by analytical calculation (Fig. 7(a), without noise) and the one measured in the experiment (Fig. 7(b)). As we can notice in Fig. 7(a), the weak and broad-spectrum acoustic wave generated by the first pulse introduces a small inaccuracy in the BFS estimation before the real hot-spot is measured. This inaccuracy has been estimated to be nearly $0.2 \mathrm{MHz}$, which is in agreement with previously reported results ${ }^{5}$. This issue can be neglected in many practical cases, since in general the introduced error is expected to be lower than the accuracy given by the system noise. As a consequence, a simple fitting of the measured BGS is enough to obtain the spatial information related to the BFS. In our experiment, the frequency resolution has been obtained calculating the standard deviation of the measured BFS, resulting in about $0.5 \mathrm{MHz}$ (representing a temperature and strain resolution of $0.5^{\circ} \mathrm{C}$ and $10 \mu \varepsilon$ ), which leads to a negligible impact of the first pulse, as reported in Fig. 7(b). The high SNR of the traces $(\sim 14 \mathrm{~dB})$ at the fiber end indicates that measurements can be extended at least up to $25-30 \mathrm{~km}$ maintaining the frequency resolution in the $1 \mathrm{MHz}$-scale.
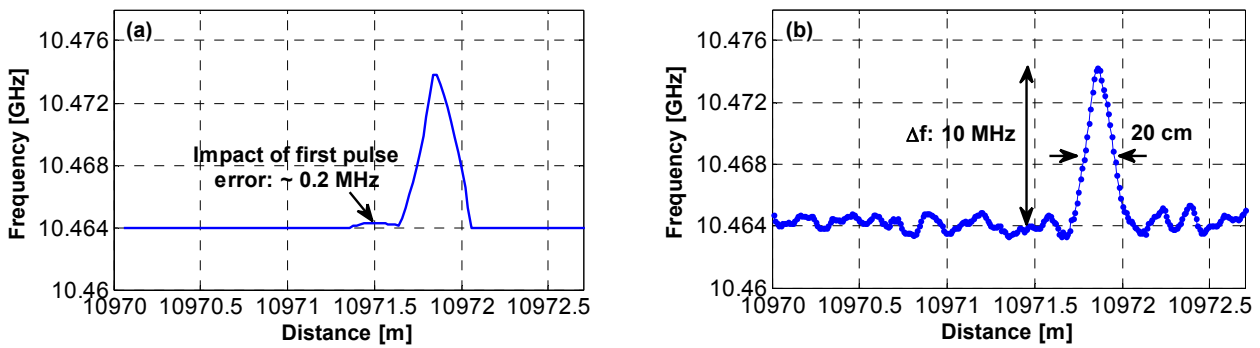

Figure $7.20 \mathrm{~cm}$ hot-spot detection at $11 \mathrm{~km}$ distance using the implemented DP-BOTDA sensor. (a) Simulated and (b) measured BFS.

In conclusion, we have proposed and experimentally validated an analytical model that explicitly describes the behavior of the acoustic wave and the probe signal in DP-BOTDA sensors. The presented analysis, based on analytical calculations and experimental results, points out that DP-BOTDA is a simple and attractive solution to achieve accurate high spatial resolution measurements, requiring a single trace acquisition and no additional devices compared to standard BOTDAs.

\section{REFERENCES}

[1] Horiguchi, T. et al., J. Lightwave Technol., 13(7), 1296-1302, 1995.

[2] Li, W., Bao, X., Li, Y, and Chen, L., Opt. Express 16(26), 21616-21625, 2008.

[3] Foaleng, S. M., Tur, M., Beugnot, J.-C., and Thévenaz, L., J. Lightwave Technol. 28 (20), 2993-3003, 2010.

[4] Kishida, K, Li, C.-H., and Nishiguchi, K, Proc. SPIE 5855, $17^{\text {th }}$ Int. Conference on Optical Fibre Sensors, 559, 2005.

[5] Koyamada, Y., IEICE Trans. Commun. E90-B(7), 1810-1815, 2007.

[6] Beugnot, J.-C., Tur, M., Foaleng, S. M., and Thévenaz, L., Opt. Express 19(8), 7381-7397, 2011.

[7] Diaz, S., Foaleng, S. M., Lopez-Amo, M., and Thévenaz, L, IEEE Sensors J. 8(7), 1268-1272, 2008. 